\title{
Fatores preditivos de adesão ao tratamento farmacológico em idosos: estudo transversal
}

O processo de envelhecimento é dinâmico e progressivo, e as alterações morfológicas e fisiológicas levam à vulnerabilidade e doenças. Para o controle e tratamento dessas doenças, a intervenção farmacológica ainda é a principal estratégia. Assim, a adesão à terapia medicamentosa é fundamental para a eficácia e segurança do tratamento. O objetivo deste estudo foi identificar preditores de adesão à terapia medicamentosa em pacientes idosos em uso de medicação contínua. Método: trata-se de um estudo transversal, descritivo e analítico que utiliza a aplicação de uma pesquisa a idosos que frequentavam o Clube da Terceira Idade Sorocaba e a Universidade da Terceira Idade. A pesquisa teve como objetivo identificar a adesão ao tratamento farmacológico através de um questionário. A análise estatística foi realizada por meio do modelo qui-quadrado para correlacionar conformidade/não conformidade. Resultados: No que se refere à adesão, pode-se observar que idosos com tratamento para hipertensão crônica $(p=0,04)$ e dislipidemia $(p=0,01)$ apresentaram maior adesão ao tratamento proposto. Pacientes idosos com maior tempo de doença $(p=0,04)$, que recebiam medicamentos em Serviço Público de Saúde $(p=0,005)$ e classes econômicas mais baixas $(p=0,02)$ apresentaram maior adesão ao tratamento. Conclusões: Os dados deste estudo sugerem que doenças crônicas como hipertensão, dislipidemia, que apresentam maior risco de morte, levam os idosos a aderir mais ao tratamento. A falta de medicamentos quando acompanhada de sintomas de doenças, mostra suas necessidades, garantindo maior adesão. A adesão ao tratamento farmacológico esteve muito mais relacionada ao quadro da doença e ao risco que ela impõe e à condição econômica do que ao comportamento do idoso.

Palavras-chave: Fatores preditivos; Conformidade com o tratamento; Idoso.

\section{Predictive factors of adherence to pharmacological treatment in the elderly: a cross-sectional study}

The aging process is dynamic and progressive, and morphological and physiological changes lead to vulnerability and diseases. For control and treatment of these diseases, pharmacological intervention is still the major strategy. Thus, adherence to drug therapy is key to the effectiveness and safety of treatment. The aim of this study was to identify predictors of adherence to drug therapy in elderly patients using continuous medication. Methods: This is a cross-sectional, descriptive and analytical study that uses the application of a survey to elderly who attended the Senior Sorocaba Club and University of the Third Age. The research aimed to identify the adherence to pharmacological treatment through a questionnaire. Statistical analysis was performed using the chi-square model to correlate compliance/noncompliance. Results: With regard to compliance, it can be seen that seniors with chronic hypertension treatment ( $p=0.04$ ) and dyslipidemia $(p=0.01)$ showed greater adherence to the proposed treatment. Elderly patients with longer disease duration $(p=0.04)$, those who received their medication from a Public Health Service $(p=0.005)$ and lower economic classes $(p=0.02)$ showed more compliance with treatment. Conclusions: The data from this study suggest that chronic diseases such as hypertension, dyslipidemia, which contain a greater risk for death, lead elderly people to comply more to the treatment. Lack of medication when accompanied by symptoms of diseases, show your needs, ensuring greater compliance. Compliance to pharmacological treatment was much more related to the disease condition and the risk it imposes as well as the economic condition than with the behavior of the elderly.

Keywords: Predictive factors; Treatment Compliance; Elderly.

\section{Topic: Enfermagem Geral}

Reviewed anonymously in the process of blind peer.
Received: 16/06/2021

Approved: 12/07/2021

Leandro Aparecido Souza

Universidade de Sorocaba, Brasil

http://lattes.cnpq.br/6090315154831086

http://orcid.org/0000-0001-8828-9918

leandroapsouza14@gmail.com

Fernando de Sá Del Fiol (iD

Universidade de Sorocaba, Brasil

http://lattes.cnpq.br/2864595160355410

http://orcid.org/0000-0002-7138-0376

fernando.fiol@prof.uniso.br

\section{Referencing this:}

SOUZA, L. A.; FIOL, S. D.. Fatores preditivos de adesão ao tratamento farmacológico em idosos: estudo transversal. Scire Salutis, v.11, n.3, p.38-45, 2021. DOI: http://doi.org/10.6008/CBPC2236$\underline{9600.2021 .003 .0006}$ 


\section{INTRODUÇÃO}

Nos últimos anos nota-se um crescimento considerável de pessoas acima de 60 anos. Segundo o Instituto Brasileiro de Geografia e Estatística (IBGE), a população idosa era de 4,8\% em 1991, passando para 5,9\% em 2000 e a 7,4\% em 2010 (IBGE, 2014). O Conselho Estadual do Idoso (2014) prevê que para 2050 a população idosa chegará em $30 \%$. Com este crescimento, há uma maior vulnerabilidade em desenvolver doenças crônicas nesta população isso requer alterações no estilo de vida e acompanhamento da evolução da doença (DIAS et al., 2011). O uso concomitante de vários medicamentos tem se tornado uma realidade constante em seu cotidiano (ROCHA et al., 2008).Com o passar do tempo, o envelhecimento e as grandes transformações desta fase implicam em mudanças de comportamentos e hábitos, tornando-o mais resistentes aos tratamentos prescritos pelo médico, buscando soluções paralelas ao seu ponto de vista, na tentativa em solucionar os problemas de saúde (MARQUES et al., 2010).

A adesão farmacológica é um dos principais determinantes da eficácia do tratamento, isso quer dizer que, quando o comportamento do paciente está de acordo com as orientações do médico ou profissional de saúde o tratamento é considerado efetivo. A adesão pode ser vista como a colaboração, cooperação, resultando-o no envolvimento de sua terapêutica (CINTRA et al., 2010), quando há entendimento por parte do paciente, e ele participa e assume responsabilidades em todo tratamento, seja medicamentoso ou não, isso faz grandes diferenças (LEITE et al., 2003). Uma das definições de adesão terapêutica é tomar o medicamento em doses e horários corretos, seguir uma dieta adequada, de acordo com o recomendado, procurar comparecer às consultas periodicamente, isso é um processo dinâmico com mudanças de comportamentos constantes (WHO, 2003), ela pode ser considerada adequada ou caracterizada como aderentes quando as prescrições e ou recomendações profissionais sejam seguidas em pelo menos, $80 \%$ de seu total (ALMEIDA et al., 2007).

Nos países desenvolvidos a adesão farmacológica dos idosos corresponde a apenas $50 \%$ dos tratados, isso reflete no aumento das hospitalizações, internações em clínicas, diminuição da qualidade de vida e aumento da morbimortalidade nesta faixa etária (SANTOS et al., 2015).

A adesão farmacológica pode ser influenciada por vários fatores, acredita-se que quanto maior o número de medicamentos o idoso utiliza, menor adesão ao tratamento, entretanto quanto maior idade, melhor adesão ao tratamento. Pacientes adultos jovens têm maior dificuldade em aceitar a condição crônica que a doença estabelece (BARBOSA et al., 2012) Acredita-se que quanto maior a idade, maior o medo em morrer, fazendo com que sejam mais adeptos à terapêutica (SGNAOLIN et al., 2012). Fatores socioeconômicos, prescrições inapropriadas também são responsáveis por grande parte de não adesão à população idosa. Acredita-se que estas situações aliadas às dificuldades de acesso aos serviços públicos e a todos os fatores já descritos, podem influenciar diretamente na qualidade de vida relacionado à saúde, e na adesão à farmacoterapia (OLIVEIRA et al., 2012). A não adesão é um problema multidimensional, onde todos estes diferentes fatores se encontram (WHO, 2003) e pode ser considerado um problema mundial por reduzir os resultados esperados em relação às doenças crônicas (MAGNABOSCO et al., 2015).

Para que exista um envelhecimento bem sucedido se faz necessário o envolvimento pessoal, 
familiar e multiprofissional, assim, haverá melhores condições para oferecer a assistência qualificada e conscientizá-los (CRUZ et al., 2011). A equipe multidisciplinar tem se mostrado importante neste processo, pois influencia positivamente na adaptação da doença e no tratamento farmacológico, cada profissional assumindo seu papel de forma clara e distinta, bem como, as prescrições médicas com dosagens e indicações corretas, isto é, uma prescrição com valor terapêutico (MONTENEGRO et al., 2014).

Considerando o elevado número de prescrições dos medicamentos de uso contínuo, bem como sua distribuição gratuita nos serviços de saúde, o levantamento destes aspectos se torna de alta a relevância para o delineamento de estratégias mais eficazes, com vistas à melhora a adesão ao tratamento (MAGNABOSCO et al., 2015). Diante do contexto apresentado, surgiu uma questão norteadora para o desenvolvimento deste estudo: “Quais os fatores preditivos a adesão farmacológica nos idosos"?

E nesta perspectiva definiu-se como objetivos do estudo identificar os fatores preditivos de adesão à terapia farmacológica em pacientes idosos sob tratamento com medicamentos de uso contínuo e traçar um perfil do idoso aderente e não aderente à terapia medicamentosa de uso contínuo.

\section{METODOLOGIA}

Tratou-se de um estudo transversal, descritivo e analítico. Foi utilizado um check list com recomendações americanas para estudos observacionais, denominado recomendações STROBE (Strengthening of Observational Studies in Epidemiology) (MALTA et al., 2010). O levantamento dos dados foi realizado no período de fevereiro a abril de 2018, na Universidade da Terceira Idade, espaço vinculado à Universidade de Sorocaba, frequentados por aproximadamente 500 idosos que semanalmente, realizam cursos de extensão. Os dados também foram coletados no "Clube do Idoso", espaço mantido pela Prefeitura Municipal de Sorocaba, que conta com aproximadamente 2.500 idosos associados, que frequentam o local semanalmente e desfrutam de diversas atividades oferecidas no local.

Foram incluídos no estudo idosos a partir de 60 anos que frequentavam as duas unidades e que fizeram uso regular de qualquer medicamento há pelo menos 3 meses. A maioria dos participantes era do sexo feminino, casados, com idade de 60 - 70 anos, com nível de escolaridade elevados, e classe social A e B. A amostragem foi aleatória, e todos que aceitaram participar da pesquisa foram incluídos, sem utilização de critérios de escolha. Foram classificados como perdas ou recusas aqueles indivíduos que, mesmo após ciência do projeto, não aceitaram em responder o instrumento. Foram excluídos aqueles que recebiam atenção de cuidadores, ou aqueles incapazes de fazer o uso do medicamento de forma autônoma. Também foram excluídos aqueles com formação profissional na área de saúde.

Foi aplicado um questionário, contendo questões com dados pessoais, socioeconômicos e questões relacionadas ao uso de medicamentos de uso contínuo, com o objetivo de identificar o perfil dos medicamentos utilizados, e os fatores preditivos à adesão do fármaco de uso contínuo. A adesão ao tratamento foi determinada por 4 questões direcionadas exclusivamente a este fim com respostas sim ou não: Quando sente se mal, você deixa de tomar o medicamento? Esquece-se de tomar o medicamento? Você é descuidado no horário em tomar o medicamento? Quando sente se bem deixa de tomar o 
medicamento? Com qualificação de aderentes para 0 e 1 resposta sim e não aderentes para 2 a 4 respostas sim. As questões de caráter sócio econômico foram utilizadas a metodologia do critério Brasil (ASSOCIAÇÃO BRASILEIRA DE EMPRESAS DE PESQUISA, 2015).

Antes do início da coleta de dados, aplicou-se um questionário piloto, com o intuito de identificar possíveis falhas. Foi realizada uma entrevista prévia com 10 idosos pelo pesquisador responsável, e houve a necessidade de reajuste no instrumento em duas questões por apresentarem confusão na interpretação. Após a aplicação do questionário piloto, essas adaptações foram feitas e o documento definitivo passou a ser aplicado.

Os idosos foram abordados individualmente ou em conjunto durante as atividades desenvolvidas nos dois espaços de coleta, sendo convidados a participar da pesquisa. Neste momento foram entregues os termos de consentimento livre esclarecido e após leitura, caso aceitassem a participar, assinavam os termos e posteriormente foram entregues os questionários para responder.

O tamanho da amostra foi calculado na proporção (Aderentes/Não Aderentes), utilizou-se da população aderentes ao tratamento (50\%), com um nível de significância de $5 \%$ com erro médio de $6 \%$ (LEVIN, 2012). A análise estatística foi por meio do software BioStat e os resultados foram considerados significativos quando o valor de $p<0,05$. As variáveis foram analisadas através de tabelas descritivas, com análise qui-quadrado para associação adesão/não adesão. O intervalo de confiança utilizado foi de $95 \%$.

O projeto foi submetido ao Comitê de Ética e Pesquisa da Universidade de Sorocaba. Os participantes foram esclarecidos quanto os objetivos da pesquisa, as estratégias de coleta de dados e assegurados quanto o sigilo, anonimato e possibilidade de interrupção da participação em qualquer momento. Os que consentiram em participar da pesquisa assinaram o termo de consentimento livre e esclarecido conforme resolução 466/2012.

\section{RESULTADOS E DISCUSSÃO}

Foram abordados 325 idosos, dos quais 20 recusaram a participação. Dos 305 que aceitaram a participação, 12 foram inseridos nos critérios de exclusão por serem profissionais da saúde e por utilizar de cuidadores para administrar os medicamentos. Desta forma, 288 idosos foram inclusos no estudo.

Os resultados mostram que $197(68,4 \%)$ dos idosos entrevistados, mostraram-se aderentes aos tratamentos empregados. A tabela 1 correlaciona os fatores socioeconômicos com a adesão ao tratamento. Os dados apresentados na tabela 1 mostram uma amostra com predominância de mulheres, abaixo de 70 anos, vivendo em companhia de esposo/esposo, parente ou cuidador. Nota-se o grande grau de escolarização dos entrevistados, com cerca de $3 / 4$ com ensino médio e superior. A grande maioria não exerce atividade remunerada. A maioria dos idosos entrevistados pertence às classes $A$ e $B$, com atendimento médico e acesso a medicamentos pela rede privada.

Importante destacar que os indicadores sócioeconomicos que guardaram relação com a adesão ao tratamento foram aqueles relacionados às questões econômicas, ou seja, nossos dados mostraram que idosos das classes C, D e E apresentaram um percentual de adesão de quase $75 \%$, com chance 1, 6 vezes 
maior $(p=0,029)$ de aderir ao tratamento do que idosos das classes A e B. No mesmo sentido, idosos que adquiriram seu medicamento no SUS apresentaram $82 \%$ de adesão ao tratamento, índice bastante superior $(p=0,005)$ dos $63,7 \%$ de adesão daqueles que referiram comprar os medicamentos para seus respectivos tratamentos. Importante ressaltar que os índices de escolarização não guardaram relação com a adesão ao tratamento farmacológico.

\begin{tabular}{|c|c|c|c|c|c|c|c|c|c|c|}
\hline & & $\mathrm{N}$ & $\%$ & Aderente & $\begin{array}{l}\text { \% } \\
\text { adesão }\end{array}$ & $\begin{array}{l}\text { Não } \\
\text { aderente }\end{array}$ & $\mathrm{p}$ & OR & IC inf & IC sup \\
\hline \multicolumn{11}{|l|}{ Sexo } \\
\hline & Feminino & 235 & $81,60 \%$ & 158 & $67,23 \%$ & 77 & & & & \\
\hline & Masculino & 53 & $18,40 \%$ & 39 & $73,58 \%$ & 14 & 0,185 & 0,737 & 0,377 & 1,438 \\
\hline \multicolumn{11}{|c|}{ 年 } \\
\hline & 59 a 69 anos & 180 & $62,50 \%$ & 122 & $67,78 \%$ & 58 & & & & \\
\hline & 70 a 79 anos & 86 & $29,86 \%$ & 61 & $70,93 \%$ & 25 & 0,302 & 0,862 & 0,492 & 1,510 \\
\hline & 80 ou mais & 22 & $7,64 \%$ & 14 & $63,64 \%$ & 8 & 0,348 & 1,202 & 0,477 & 3,026 \\
\hline \multicolumn{11}{|c|}{ Estado civil } \\
\hline & Casado & 152 & $52,78 \%$ & 103 & $67,76 \%$ & 49 & & & & \\
\hline & Divorciado/Solteiro/Viúvo & 136 & $47,22 \%$ & 94 & $69,12 \%$ & 42 & 0,403 & 0,939 & 0,571 & 1,546 \\
\hline \multicolumn{11}{|c|}{ Com quem reside } \\
\hline & Companhia & 227 & $78,82 \%$ & 152 & $66,96 \%$ & 75 & & & & \\
\hline & Sozinho & 61 & $21,18 \%$ & 45 & $73,77 \%$ & 16 & 0,155 & 0,721 & 0,382 & 1,359 \\
\hline \multicolumn{11}{|c|}{ Escolarização } \\
\hline & Fundamental + funcional & 76 & $26,39 \%$ & 54 & $71,05 \%$ & 22 & & & & \\
\hline & Médio + Superior & 212 & $73,61 \%$ & 143 & $67,45 \%$ & 69 & 0,281 & 1,184 & 0,668 & 2,101 \\
\hline \multicolumn{11}{|c|}{ Exerce atividade remunerada } \\
\hline & Não & 233 & $80,90 \%$ & 161 & $69,10 \%$ & 72 & & & & \\
\hline & Sim & 55 & $19,10 \%$ & 36 & $65,45 \%$ & 19 & 0,301 & 1,180 & 0,634 & 2,197 \\
\hline \multicolumn{11}{|c|}{ Classe econômica } \\
\hline & $C$, D e E & 115 & $39,93 \%$ & 86 & $74,78 \%$ & 29 & & & & \\
\hline & $A$ e $B$ & 173 & $60,07 \%$ & 111 & $64,16 \%$ & 62 & 0,029 & 1,656 & 0,982 & 2,796 \\
\hline \multicolumn{11}{|c|}{ Fonte do medicamento } \\
\hline & SUS & 56 & $19,44 \%$ & 46 & $82,14 \%$ & 10 & & & & \\
\hline & Comprado & 168 & $58,33 \%$ & 107 & $63,69 \%$ & 61 & 0,005 & 2,622 & 1,235 & 5,568 \\
\hline & Parte SUS parte comprado & 65 & $22,57 \%$ & 45 & $69,23 \%$ & 20 & 0,505 & 2,044 & 0,862 & 4,848 \\
\hline \multicolumn{11}{|c|}{ Em que local é atendido } \\
\hline & SUS & 99 & $34,38 \%$ & 71 & $71,72 \%$ & 28 & & & & \\
\hline & Particular & 234 & $81,25 \%$ & 162 & $69,23 \%$ & 72 & 0,326 & 1,127 & 0,671 & 1,892 \\
\hline
\end{tabular}

Tabela 1: Distribuição dos idosos participantes de acordo com análise descritivas da associação entre adesão ao tratamento com o perfil da população e fatores socioeconômicos, Sorocaba, SP, Bras

Os resultados da tabela 2 mostram que os portadores de doenças crônicas como dislipidemia e hipertensão apresentaram um percentual de adesão $(72,2 \%$ e 78,6\%) muito superior $(p=0,042$ e 0,013) aos não portadores das respectivas doenças. Com relação ao tempo de doença crônica, os idosos com doença crônica há mais de 3 anos apresentaram uma adesão ao tratamento maior (70\%) do que aqueles com até 3 anos de diagnóstico da doença crônica. De todos os cruzamentos referentes às questões ligadas à saúde dos idosos, chama a atenção o baixo grau de adesão (50\%) daqueles que referiram necessitar de outra pessoa para lembrar-se de usar o medicamento. A adesão de $50 \%$ é significativamente menor $(p=0,02)$ daqueles que referiram utilizar a memória para a utilização correta do medicamento.

A prevalência de indivíduos aderentes ao tratamento farmacológico identificada neste estudo foi maior que 50\%, visto que Santos et al. (2015) declara que nos países desenvolvidos a média de adesão farmacológica corresponde a 50\%.Talvez esta diferença possa estar relacionada ao grau de escolaridade médio + superior dos idosos de $67 \%$, ou seja uma população com nível de graduação e fatores socioeconômicos elevados, apesar destes dados, isso não demonstrou significância estatística junto ao 
desfecho de interesse.

Tabela 2: Distribuição dos idosos participantes de acordo com análise descritivas da associação entre adesão ao tratamento com os fatores de saúde, Sorocaba, SP, Brasil, 2018.

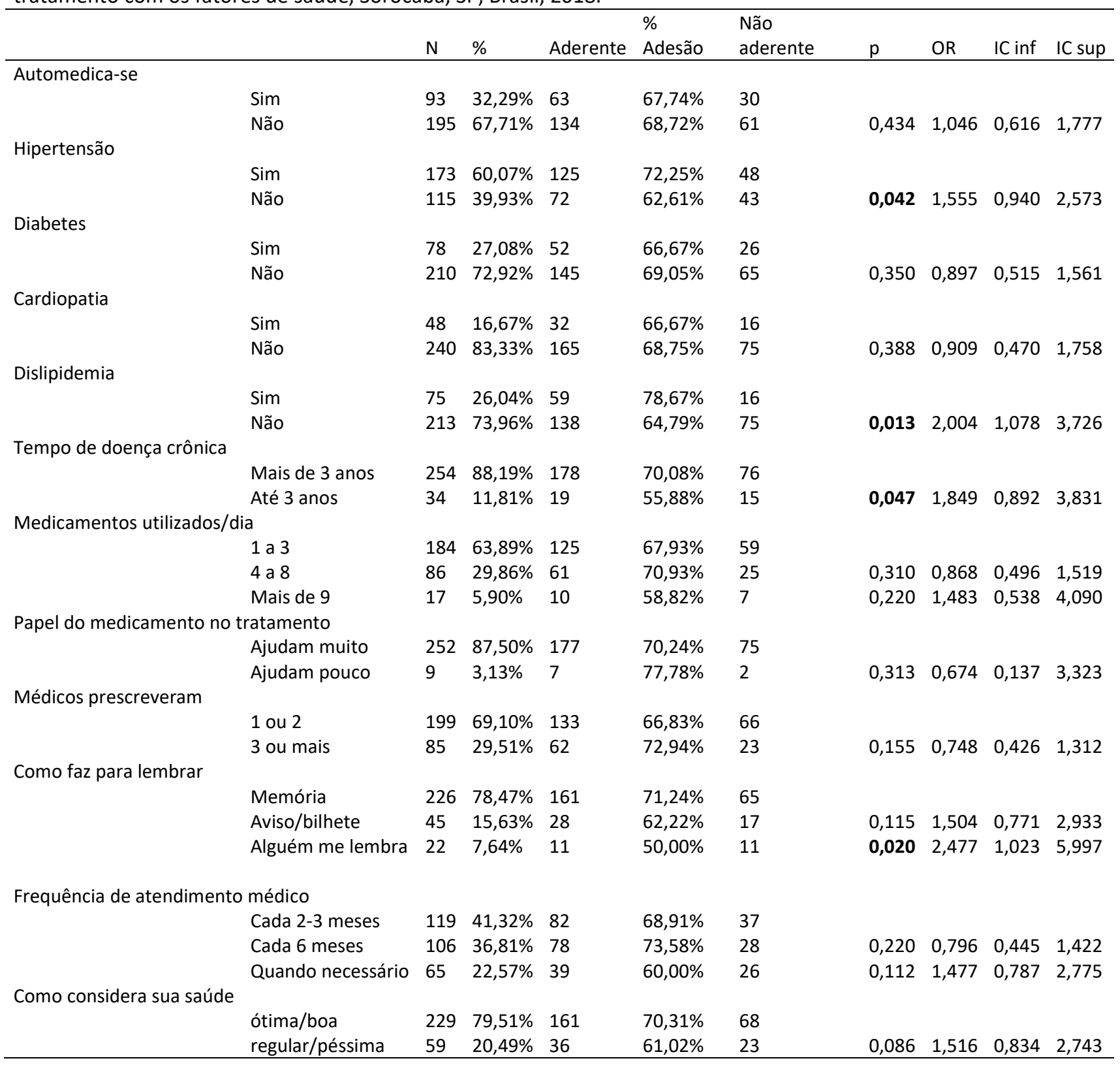

As variáveis gênero, idade, não demonstraram significância estatística neste estudo, Sgnaolin et al. (2012), defende que quanto maior idade, maior adesão. Já Barbosa et al. (2012), acreditam que adultos jovens têm maiores dificuldades em aceitar a condição crônica que a doença estabelece, fazendo com que a adesão seja menor. As proporções são equivalentes quando temos idosos que residem sozinhos/solteiros/viúvos comparados com os casados e que não residem sozinhos. Cruz et al. (2011) descreve que se faz necessário o envolvimento familiar para melhor conscientizá-los à importância da adesão.

As classes sociais C, D e E correspondem a $74 \%$ e são consideradas aderentes, com nível de significância com $p<0,05$, onde a chance destas classes serem adeptas ao tratamento são 0,6 vezes, com IC $95 \%(0,98-2,76)$. O local de atendimento destes idosos foram na grande maioria em consultórios particulares/convênios com $81 \%$, em contrapartida os idosos que retiram os seus medicamentos no SUS 
tem se mostrado mais adeptos ao tratamento com $p=0,005$. Neste caso confirmam a informação de Oliveira et al. (2012) que os fatores socioeconômicos influenciam na adesão terapêutica. Os indivíduos que retiram os medicamentos exclusivamente nos serviços públicos de saúde, tem uma relação forte com a adesão, com $p<0,05$ com uma chance de 1,6 vezes serem mais aderentes ao tratamento. Isso nos diz que os idosos que não compram os medicamentos estão mais envolvidos à terapêutica medicamentosa. Oliveira et al. (2012), descreve que as dificuldades de acesso aos serviços públicos, podem influenciar diretamente na adesão à farmacoterapia.

Os idosos que relataram ser hipertensos e ou dislipidêmicos são mais aderentes com grau de significância de $p=0,04$ e 0,01 respectivamente, comparados com os idosos que são diabéticos e ou cardiopatas. Parece que os sinais e sintomas imediatos que a hipertensão causa na ausência dos medicamentos faz com que o idoso não se esqueça de tomar o medicamento, tornando o tratamento mais efetivo. Quanto à dislipidemia acredita-se que o medo em ter uma complicação mais grave na doença de base, ou até morrer faz os indivíduos colaborarem mais com o tratamento (SGNAOLIN et al., 2012).

A quantidade dos medicamentos utilizados pelos idosos são em média 3 tipos de medicamentos diários, e sua auto percepção do quanto o medicamento lhe ajudam em sua vida aproxima-se de $90 \%$ dos que responderam ajudam muito no processo saúde doença, mas sem significância estatística. 0 tempo correspondente da doença, interfere no processo de adesão, pois como vemos nestes resultados que pessoas com diagnóstico com tempo maior que 3 anos são mais aderentes em comparação aos recém diagnosticados, com uma chance de 0,8 vezes e IC $95 \%(0,89-3,83)$. Apesar de existirem inúmeras formas para lembrar-se de tomar os medicamentos, a maioria dos idosos declararam guardar na memória, e estes têm maior adesão aos que utilizam de algo ou alguém para lembrá-los, com p=0,02.

\section{CONCLUSÕES}

Apesar das limitações apresentadas no estudo, com a presença de idosos com níveis de escolaridade elevados, e fatores socioeconômicos satisfatórios, podemos concluir este estudo que poucos são os fatores preditivos utilizados para garantir a melhor adesão farmacológica nesta população.

O perfil da população aderente são os idosos jovens, entre 60 a 69 anos, com doenças crônicas como a hipertensão arterial sistêmica e as dislipidemias, os que tem o diagnóstico medico da doença há um tempo maior que 3 anos, e aqueles que utilizam da memória para lembrar-se de tomar os medicamentos. Em relação aos fatores socioeconômicos podemos afirmar que a classe econômica C,D e E são mais aderentes ao tratamento medicamentoso, assim como aqueles que utilizam dos serviços públicos para aquisição de seus medicamento.

\section{REFERÊNCIAS}

ALMEIDA, H. O.; VERSIANI, E. R.; DIAS, A. R.; NOVAES, M. R. C. G.; TRINDADE, E. M. V.. Adesão a tratamentos entre idosos. Revista Brasileira de Ciências e Saúde, v.18, n.3, p. 57-67, 2007.

BARBOSA, B. R. G.; FERRIOLLI, E.; MORIQUITI, J. C.; NOGUEIRA, C. B.; NOBRE, F.; UETA, J.; LIMA, N. K.. Treatment adherence and blood pressure control in older individuals whith hypertension. Arquivos Brasileiros de Cardiologia, v.99, n.1, p.636-640, 2012. DOI: https://doi.org/10.1590/S0066-782X2012005000054

CINTRA, F. A.; GUARLENTO, M. E.; MIYASAKI L. A.. Adesão medicamentosa em idosos em seguimento ambulatorial. 
Revista Ciências \& Saúde Coletiva, v.15, n.3, p.3507-3515, 2010. DOI: https://doi.org/10.1590/S1413$\underline{81232010000900025}$

CRUZ, L. P.; MIRANDA, P. M.; VEDANA, K. G. G.; MIASSO, A. I.. Terapêutica medicamentosa: adesão, conhecimento e dificuldades de idosos em transtorno bipolar. Revista Latino Americana de Enfermagem, v.4, n.19, p.1-9, 2011. DOI: https://doi.org/10.1590/S0104-11692011000400013.

DIAS, J. A.; ARREGUY-SENA, C.; PINTO, P. F.; SOUZA, L. C.. Ser idoso e o processo de envelhecimento: saúde percebida. Escola Anna Néri Revista de Enfermagem,v.2, n.15, p.372379, 2011. DOI: https://doi.org/10.1590/S1414$\underline{81452011000200021}$

IBGE. Instituto Brasileiro de Geografia e Estatística. Cidades. IBGE, 2018.

LEITE, S. N.; VASCONCELLOS, M. P. C.. Adesão à terapêutica medicamentosa: elementos para discussão de conceitos e pressupostos adotados na literatura. Revista Ciências \& Saúde Coletiva, v.8, n.4, p.775-782, 2003. DOI: https://doi.org/10.1590/S1413-81232003000300011

LEVIN, J.; FOX, J. A.; FORDE, D. R.. Estatística Aplicada a Ciências Humanas. 11 ed. São Paulo: Pearson, 2012.

MAGNABOSCO, P.; TERAOKA, E. C.; OLIVEIRA, E. M.; FELIPE, E. A.; FREITAS, D.; ALVES, L. M. M.. Análise comparativa da não adesão ao tratamento medicamentoso da hipertensão arterial sistêmica em população urbana e rural. Revista Latino Americana de Enfermagem, v.23, n.1, p.20-27, 2015. DOI: https://doi.org/10.1590/0104-1169.0144.2520

MALTA, M.; CARDOSO, L. O.; BASTOS, F. I.; MAGNANINI, M. M. F.; SILVA, C. M. F. P.. Iniciativa STROBE: subsídios para a comunicação de estudos observacionais. Revista Saúde Pública, v.44, n.3, p.559-565, 2010.

MARQUES, E. I. W.; PETUCO, V. M.; GONÇALVES, C. B. C.
Motivos da não adesão ao tratamento médico prescrito entre os idosos de uma unidade de saúde da família do município de Passo Fundo-RS. Revista Brasileira de Ciências do Envelhecimento Humano, v.7, n.2, p.267-279, 2010. DOI: https://doi.org/10.5335/rbceh.2012.865

MONTENEGRO, A. J. N.; LUQUE, A. M.; AURIOLES, E. M.; VERDU, B. T.; MORENO, C. L.; CORREA, J. A. G.. Adherencia al tratamiento en pacientes polimedicados mayors de 65 años com prescripcion por princípio activo. Revista Atencion Primária, v.46, n.5, p.238-245, 2014.

OLIVEIRA, M. P. F.; NOVAES, M. R. C. G.. Uso de medicamentos por idosos de instituições de longa permanência, Brasília- DF, Brasil. Revista Brasileira de Enfermagem, v.5, n.65, p.737-744, 2012. DOI: http://dx.doi.org/10.1590/S0034-71672012000500004

ROCHA, C. H.; OLIVEIRA, A. P. S.; FERREIRA, C.; FAGGIANI, F. T.; SCHROETER, G.; SOUZA, A. C. A.; DECARLI, G. A.; MORRONE, F. B.; WERLANG, M. C.. Adesão à prescrição médica em idosos de Porto Alegre, RS. Revista Ciências \& Saúde Coletiva, v.13, n.1, p.703-710, 2008. DOI: http://dx.doi.org/10.1590/S1413-81232008000700020

SANTOS, W. A.; PITEIRA, A. R. S.; FERNANDES, L. F. G. B.; LEITE, B. S.; CAVALEIRO, A. J. B. G.; VALENTE, G. S. C.. Aging and therapeutic adherence as care focus on education in nursing international student exchange. Journal of Nursing UFPE on line, v.2, n.9, p.875-886, 2015. DOI: http://dx.doi.org/10.5205/reuol.6391-62431-2ED.0902supl201514

SGNAOLIN, V.; FIGUEIREDO, A. E. P. L.. Adesão ao tratamento farmacológico de pacientes em hemodiálise. Jornal Brasileiro de Nefrologia, v.34, n.2, p.109-116, 2012. DOI: https://doi.org/10.1590/S0101-28002012000200002

WHO. World Health Organization. Adherence to long term therapies, Evidence for action. WHO, 2003.

A CBPC - Companhia Brasileira de Produção Científica (CNPJ: 11.221.422/0001-03) detém os direitos materiais desta publicação. Os direitos referem-se à publicação do trabalho em qualquer parte do mundo, incluindo os direitos às renovaç̃ões, expansões e disseminações da contribuiç̃o, bem como outros direitos subsidiários. Todos os trabalhos publicados eletronicamente poderão posteriormente ser publicados em coletâneas impressas sob coordenação da Sustenere Publishing, da Companhia Brasileira de Produção Científica e seus parceiros autorizados. Os (as) autores (as) preservam os direitos autorais, mas não têm permissão para a publicação da contribuição em outro meio, impresso ou digital, em português ou em tradução. 